I.

\title{
INTRODUCTION
}




\section{THOUGHTS ABOUT VERY LARGE TELESCOPES}

\section{E.J. Wampler, Lick Observatory}

\section{Introduction}

It is perhaps useful, at the beginning of a conference such as this, to review briefly our goals and the requirements that these goals will place on our instruments. I confess to a strong personal bias in my choice of subjects but perhaps by now my beard is sufficiently gray that the community will only smile at my prejudices. Telescopes have historically been so versatile and the universe so rich in research possibilities that grave design errors have been forgiven in the face of the new knowledge that even a poorly designed or maintained telescope produces. But observational astronomy is becoming more competitive. Many nations are now willing to spend comparatively large sums of money on astronomy. Optical astronomy from space will soon begin to challenge the optical and infrared capabilities of ground telescopes. A super giant telescope will be costly to build and operate. We can no longer afford to make costly errors. We have learned a lot from the past decade and a half of large telescope construction and operation and must apply this knowledge to the design and construction problems that face us. I am optimistic about our abilities to extend past experience and produce large telescopes with sharply expanded capabilities when compared to existing instruments. We only need to be very clear about our goals and the rationale for choosing a particular aperture, configuration or design.

\section{What do we want?}

It seems to me that the most important requirement of a large telescope is image quality and stability. Aperture is secondary to this. The giant telescopes of the future will usually be operating in a sky limited mode. When sky limited the signal-to-noise ratio of the data improves inversely as the image diameter but only as the square root of the telescope mirror area. This fact, together with pessimistic estimates of the image quality has, in the past, been used to argue that the construction of a large array of small telescopes is preferable to the construction of one or a few much larger instruments. However, in my view, there is now substantial evidence that on good sites the natural seeing is frequently sub-arc second. This, together with the fact that a large telescope will be diffraction limited in the infrared during periods of good seeing, argues for telescope apertures of approximately 10 meters. The exact

Proceedings of the IAU Colloquium No. 79: "Very Large Telescopes, their Instrumentation and Programs", Garching, April 9-12, 1984. 
aperture chosen depends on the confidence of the designers in being able to maintain unprecedented optical quality in the presence of wind loading and thermal and support deformations.

There is now considerable evidence that from an optical point of view domes are bad things (Woolf and Ulich, 1983). Domes have been justified by the need to protect the telescope from the weather and shelter it from wind buffeting. But they are big and expensive. Their size tempts designers to install sleeping quarters, kitchens, lab space etc. in the dome. Air escaping into the dome from these warm areas hinders the advancement of astronomical knowledge by destroying the seeing. It is not simple to design a telescope that is stiff against wind loading in an exposed environment; but $I$ suspect that it would be cheaper to build a stiff telescope with a roll off weather shed than to build a flimsy telescope and house it in a conventional dome. More studies of the influence of the telescope structure and dome on the seeing are needed.

A second important requirement for a large telescope is field of view. While in principle the information transfer capability of an optical system is the product of the aperture and field of view a wide field of view is only useful if it can be used. In the past large fields were only used for photography. But in the future it can be expected that multi-aperture spectroscopy using fiber optic feeds will become increasingly important. A large telescope is needed to reduce the integration time sufficiently that differential atmospheric refraction does not move the relative separations of the objects more than the acceptance aperture of the fibers. I don't intend here to discuss the scientific programs of the large telescopes. But to illustrate the situation it is worth mentioning two specific programs for a multifiber spectrograph that would require large fields. UVX and objective prism surveys of quasars have now produced far more candidate objects than can reasonably be studied by telescopes equipped with only on-axis spectrographs. At the fainter magnitudes there are tens to hundreds of quasars per square degree. The accurate spectrophotometry of the many objects needed for meaningful statistical studies can only be obtained by observing candidate objects simultaneously. A second major program for multi-aperture spectroscopy will be the study of faint galaxy clusters and superclusters. The nearer groups might cover a substantial fraction of a degree while the more distant ones would cover a few tens of arc minutes. The collection of the thousand or so spectra needed to separate group members from field objects would be an impossible task if the spectra are obtained one at a time.

The natural sky background is an important consideration. Obviously it is important to avoid locations that are subject to intense auroral activity. 
Yet satellite studies of the night airglow show that there are regular patterns over the earth's surface (E.P. Ney, private communication). These patterns seem to be more or less fixed geographically. It is important to find areas that are relatively quiet. The local albedo of the ground can also influence the sky background by reflecting moon or starlight back through the atmosphere. On site measurements of the airglow and its correlation with solar activity is important for site evaluation.

The requirements listed above are strongly zenith distance dependent. At zenith angles exceeding about $45^{\circ}$ the optical performance of the telescope is increasingly compromised. The natural conclusion is that the mechanical designers should optimize the telescope performance for the zenith. It also suggests that a number of telescopes at different geographical latitudes would be required for complete sky coverage. This is a happy situation. There is need for all the large telescopes that are now being planned! Near the zenith the telescope should have very tight performance requirements for collimation and focus stability, thermal deformations, flutter under wind loading, pointing accuracy and tracking smoothness. In practice these "mechanical" problems can decrease the sensitivity of the instrument as much as poor mirror figure.

For infrared work the telescope must have low thermal background. The telescope pupil should be thermally stable and sufficiently simple that it can be easily masked. The telescope should be diffraction limited at the Ionger $(10 \mu \mathrm{m})$ wavelengths because it is to be expected that at good sites the seeing conditions will occasionally allow diffraction limited operation.

The pointing and tracking ability of the telescope should be good enough to allow the telescope to be operated in a "blind" mode. Blind pointing would be needed for possible daytime IR observations. Even at night there will be occasions, as when observing dark galactic clouds in moonlight, when no bright guide stars will be present in the field. However in general rather bright $(\mathrm{m}<14 \mathrm{mag})$ guide stars with accurate positions will be available. The Space Telescope Institute is in the process of measuring the positions and magnitudes of a large number of stars to be used to guide the space Telescope. These stars will define an accurate, stable reference system that could be used by ground based telescopes for stabilizing the pointing, focus and collimation.

The enormous information collecting capability of the giant telescopes together with the strong bias for zenith work during conditions of good seeing suggests that the telescope should be designed for rapid instrument change. Program queuing with frequent program changes rather than full nights given to individual astronomers seems sensible. On line data reduction, at least to the "quick look" stage and remote terminals, perhaps located on a different 
continent than the telescope, naturally flow from this novel mode of operation. All this implies that there must be rather extensive computational capability near the telescope site. These computing facilities need to be maintained and this maintenance may be expensive at a remote site.

\section{Management}

The large telescopes that have been built in the United States have generally been designed by a small group led by one, or at the most, a few, strong individuals. They have not been "committee telescopes". The prototype of such efforts is the Hale 5-meter telescope on Mt. Palomar. A large number of individuals contributed their ideas and effort but the group formed around G.E. Hale and was held together by his vision of the giant telescope.

The construction of any telescope of the size of the ones under consideration at this conference will generate a seemingly endless string of problems, each of which must be solved for the telescope to work. One person, or even a small group, cannot solve all the problems in a timely fashion. Thus the design of the telescope and the management of the construction phase must necessarily involve a large group of engineers and technicians. Because the telescope must be designed to meet the needs of the user community there must be open communication channels between the user groups and the design team. For a broad national or an international effort these communication channels may become quite complex.

Although the performance goals of the telescope should be arrived at by consensus with the user group and the expeditious solution of the technical problems will require a large technical group it will be necessary for a strong, central leadership to set the "tone" or "style" of the telescope. The design of these giant telescopes must break new ground if the costs are to remain reasonable in the face of very demanding performance goals. A management committee would find many areas for disagreement and would most likely generate costly delays in the design and construction of the telescope. Central leadership has the advantage that it is likely to be sufficiently biased to make decisions and cheerfully bear the responsibility for its decisions. This feeling of responsibility is an important ingredient in the commissioning of the telescope. The success of the 200-inch telescope is not only the result of good design but also is due to the great effort of Ira S. Bowen who made the telescope "work" after it was "finished". 
IV. Technology Development

The "buzz word" in large telescope circles is "new technology". I think this term is so loosely defined as to be almost meaningless. Its frequent use seems to me to only confuse the design of low cost, high performance telescopes. It might be useful to carefully define our meanings. It seems to me that by "new technology" people really mean three quite different and separate things. I would classify these as:

a. Adaptive technology - this is the application of quite well understood knowledge to the problems encountered in the construction of a large telescope. Examples would include: computer controls, finite element analysis of structures and mirror support systems, glass fiber feeds for spectrographs, modern servo systems etc.

b. Exploratory technology - here I mean the study of systems that exist but whose properties are insufficiently understood to be confident that their application would represent a solution to a particular large telescope problem. Examples include: the thermal and strain properties of large glass or metal mirrors. The life and hysteresis properties of screw actuators. The characteristics of glue joints. The impact of a large telescope and dome on the local seeing etc.

c. Development or emerging technology - this term is reserved for possible solutions that require substantial development before they could be usefully applied. Examples would include: composite glass or fiber materials for mirrors. Broad band super reflective coatings for large mirrors. Infrared detector arrays etc.

The difficulty and risk of the technology increases as we move from a to c. We can be thankful that most of the technology needed for a large telescope can be listed under a. The hallmark of an innovative yet conservative approach is that the selected solution works and is forgiving to changes in the parameters of its environment. If one has great difficulty in making a solution work at all it is likely that the chosen solution will be difficult to maintain in the field. Then the performance of the telescope will inevitably decline. An example is the right ascension arive worm for the Lick observatory 120-inch Shane Telescope. Much difficulty was experienced in making and lapping the gears initially. After 25 years of operation the R.A. drive worm gear has several hundred microns of wear and has developed a 2 arc second periodic error. The similar slew worm gear has very little wear. Since the telescope can only rotate $\pm 180^{\circ}$ the slew worm must have had at least as much travel as the R.A. drive worn. The difference is the rate of travel. Evidently worm gears are 
subject to excessive wear if they are driven slowly.

In summary, it is worth reminding ourselves what it is that we want to do. We must remember that the acheiveable science is only weakly dependent on the exact aperture of the telescope but that many of the problems of design, execution and, finally, cost of a large telescope are sharply dependent on aperture. As one approaches the largest realizable aperture not only the construction costs but also the operating costs will steeply increase while the reliability decreases. We want a large telescope, but not one that is too big. It is better to build a somewhat smaller instrument that performs well than to have a telescope which pushes the limits of technology in several areas at once.

\section{References}

Woolf, N.J. and Ulich, B.I. 1984, Proc. ESO Workshop on Site Testing for Future Large Telescopes (ESO: Garching bei München). 